\title{
ITALIENISCHE HUMANISTEN AN DER UNIVERSITÄT WIEN IM 15. JAHRHUNDERT ZWISCHEN FÖRDERUNG UND ABLEHNUNG
}

\author{
DANIEL LUGER
}

\section{ITALIAN HUMANISTS AT THE UNIVERSITY OF VIENNA IN 15TH CENTURY - BETWEEN SUPPORT AND REJECTION}

The study focuses on a subject that has, as yet, received little scholarly attention: the humanist reform attempts at the University of Vienna from the mid-1450s until the appointment of Conrad Celtis as professor in 1497. Unprinted files are available as source material from the faculty of arts, allowing for a re-evaluation of the supposed conflict between a seemingly conservative faculty of artists and 'modern' humanist poets as well as between representatives of an assumed Italian or German humanism.

Keywords: Humanism - University of Vienna - Late Middle Ages - Emperor Friedrich III

DOI: $10.14712 / 23365730.2020 .16$

Die Diffusion des italienischen Renaissance-Humanismus nördlich der Alpen muss sicherlich als einer der einflussreichsten geistesgeschichtlichen Transferprozesse des späten Mittelalters betrachtet werden. ${ }^{1}$ Diese vielschichtige Entwicklung wird für das Gebiet der habsburgischen Erblande im Allgemeinen bzw. der Wiener Rudolfina im Besonderen von der literatur- und werkgeschichtlich dominierten Forschung ${ }^{2}$ in erster Linie mit zwei

1 Dazu allgemein Johannes Helmrath, Wege des Humanismus. Studien zur Praxis und Diffusion der Antikeleidenschaft im 15. Jahrhundert. Ausgewählte Aufsätze, I, Tübingen 2013 (Spätmittelalter, Humanismus, Reformation 72).

2 Zum Folgenden vgl. etwa die Überblicksdarstellungen von Joseph AschBACH, Geschichte der Wiener Universität, I-II, Wien 1865/77; Helmuth GrössING, Die Wiener Universität im Zeitalter des Humanismus von der Mitte des 15. bis zur Mitte des 16. Jahrhunderts, in: Günther Hamann - Kurt Mühlberger - Franz Skacel (Hgg.), Das alte Universitätsviertel in Wien 1385-1985, Wien 1985, S. 37-45 (Schriftenreihe des Universitätsarchivs 2); Kurt Mühlberger, Zwischen Reform und Tradition. Die Universität Wien in der Zeit des Renaissance-Humanismus und der Reformation, Mitteilungen der Österreichischen Gesellschaft für Wissenschaftsgeschichte 15, 1995, S. 13-42; Karl Grossmann, Die Frühzeit des Humanismus in Wien bis zu Celtis Berufung 1497, Jahrbuch für Landeskunde von Niederösterreich 22, 1929, S. 152-325; Alfred A. STRNAD, Die Rezeption von Humanismus und Renaissance in Wien, in: Winfried Eberhard - Alfred A. Strnad (Hgg.), Humanismus und Renaissance in Ostmitteleuropa vor der Reformation (Forschungen und Quellen zur Kirchen- und Kulturgeschichte Ostdeutschlands 28), Köln u. a. 1996, S. 71-135; Alfred A. STRnAD, Die Rezeption der italienischen Renaissance in den österreichischen Erbländern der Habsburger, in: Georg Kauffmann (Hg.), Die Renaissance im Blick der Nationen Europas (Wolfenbütteler Abhandlungen zur Renaissanceforschung 9), Wiesbaden 1991, S. 135-226. Einen breiteren Zugang zu dieser Thematik bieten die Beiträge in: Thomas MAISEL - Meta Niederkorn-Bruck - Christian Gastgeber (Hgg.), Artes - Artisten - Wissenschaft: die Universität Wien in 
zentralen Entwicklungsschritten verbunden: Zunächst kommt dem als „Apostel des Humanismus nördlich der Alpen“ bezeichneten Enea Silvio Piccolomini, dem späteren Papst Pius II., eine besondere Bedeutung zu. Während seiner Zeit am habsburgischen Hof, als Kanzlist und Sekretär des römisch-deutschen Königs Friedrich III., trat der Sieneser Humanist auch zwei Mal an der Wiener Universität auf und propagierte bei dieser Gelegenheit Nutzen und Vorzüge der humanistischen Bildungsbewegung. ${ }^{3}$ Die historische Forschung stellte in diesem Zusammenhang fest, dass der Italiener zwar durchaus das Interesse einiger nordalpiner Gelehrter an der Universität sowie auch am habsburgischen Hof gewinnen konnte, zu einer dauerhaften Verankerung des Humanismus in Österreich sei es zu diesem Zeitpunkt allerdings noch nicht gekommen. Die Zeit nach Piccolominis Rückkehr nach Italien ab der Mitte der 1450er Jahre wird daher in diesem Zusammenhang als Phase des Stillstandes oder gar des Rückschrittes gesehen. Nach Alphons Lhotsky seien die Österreicher „im tiefsten ,gotische" Menschen [geblieben], auch wenn sich dann der eine oder andere ein wenig antikisch drapiert". ${ }^{4}$

Die als überwiegend traditionell-scholastisch charakterisierte Rudolfina des 15. Jahrhunderts sei der italienischen Bildungsbewegung im Wesentlichen lange Zeit reserviert bis ablehnend gegenübergestanden; der Durchbruch sei dieser Geistesströmung gleichsam ex nihilo unter massivem Druck des neuen Landesherrn Maximilian I., der in seiner Jugend möglicherweise selbst eine humanistische Bildung genossen habe, ${ }^{5}$ durch die Gründung des Collegium Poetarum et Mathematicorum durch Konrad Celtis gelungen. ${ }^{6}$

Im Fokus dieses Beitrages steht der Zeitraum zwischen der Rückkehr Piccolominis nach Italien und der Berufung von Konrad Celtis nach Wien im Jahr 1497, der von der Humanismus-Forschung in Österreich bislang kaum näher untersucht wurde. An Quellenmaterial stehen in erster Linie die aus der universitären Selbstverwaltung erwachsenen Akten der Rudolfina, insbesondere der örtlichen Artisten-Fakultät, zur Verfügung. ${ }^{7}$

Spätmittelalter und Humanismus, Wien 2015 (Singularia Vindobonensia 4), sowie Laetitia Bовнм, Humanistische Bildungsbewegung und mittelalterliche Universitätsverfassung. Aspekte zur frühneuzeitlichen Reformgeschichte der deutschen Universitäten, in: Jozef Ijsewijn - Jacques Paquet (Hgg.), The Universities in the Late Middle Ages, Louvain 1978, S. 315-346 (Mediaevalia Lovaniensia 6), bzw. Walter RüEGG, Das Aufkommen des Humanismus, in: Walter Rüegg (Hg.), Geschichte der Universität in Europa, I, Das Mittelalter, München 1993, S. 387-408.

3 Zur Person Enea Silvio Piccolominis und dessen Wirken in Österreich siehe Martin Wagendorfer, Eneas Silvius Piccolomini und die Wiener Universität. Ein Beitrag zum Frühhumanismus in Österreich, in: Franz Fuchs (Hg.), Enea Silvio Piccolomini nördlich der Alpen (Pirckheimer-Jahrbuch für Renaissance- und Humanismusforschung 22), Wiesbaden 2008, S. 21-52; Johannes Helmrath, Vestigia Aeneae imitari. Enea Silvio Piccolomini als „Apostel“ des Humanismus. Formen und Wege seiner Diffusion, in: Johannes Helmrath - Ulrich Muhlack - Gerrit Walther (Hgg.), Diffusion des Humanismus. Studien zur nationalen Geschichtsschreibung europäischer Humanisten, Göttingen 2002, S. 99-141 (mit umfangreicher Bibliographie).

4 Alphons Lнотsку, Aeneas Silvius und Österreich, in: Hans Wagner - Heinrich Koller (Hgg.), Alphons Lhotsky, Aufsätze und Vorträge 3, München 1972, S. 26-71, hier S. 58. Vgl. etwa auch A. A. STRNAD, Rezeption der italienischen Renaissance, S. 155: „Die drei Jahrzehnte nach 1460 [...] waren [...] der Rezeption von Renaissance und Humanismus auf breiterer Ebene nicht sonderlich günstig“. Ein etwas ausgeglichenes Urteil bei K. Grossmann, Frühzeit, S. 258 und 268.

5 Siehe dazu zuletzt Daniel Luger, Eine Erziehung im Geist des Humanismus? Johannes Hinderbach und die Lehrer des jungen Maximilian, in: Katharina Kaska (Hg.), Kaiser Maximilian I. Ein großer Habsburger, Wien 2019, S. 36-45.

6 Vgl. allerdings jüngst das ausgewogene Urteil von Christian LACKNER, Maximilian und die Universität Wien, in: Katharina Kaska (Hg.), Kaiser Maximilian I. Ein großer Habsburger, Wien 2019, S. 46-55.

7 Ich bedanke mich bei dieser Gelegenheit insbesondere bei Doz. Johannes Seidl (Universitätsarchiv Wien) für zahlreiche Hinweise und Hilfestellungen. Zu Funktion und Aussagewert dieser Quellengattung samt 
Bereits ein Blick auf die in den Fakultätsakten verzeichnete Zusammensetzung und Entwicklung des universitären Bücherbestandes mahnt zur Vorsicht gegenüber einer grundsätzlichen Widerständigkeit der Universität Wien gegenüber dem italienischen Humanismus. ${ }^{8}$ So vermerkt etwa für den 21. Oktober 1467 der Dekan der artistischen Fakultät, Wolfgang Hayden, das Erscheinen eines Kaufmannes aus Verona mit humanistischen und historischen Büchern (libros venales artem humanitatis et hystorias tocius mundi respicientes). Die Fakultät habe diese Gelegenheit gerne genutzt und ad perpetuum decus facultati 27 Bücher, die als ausgesprochen nützlich erachtet wurden, für 90 Dukaten erworben, darunter u.a. Werke von Vergil, Sallust, Cicero, Seneca, Terenz und Horaz sowie Texte von Humanisten wie Francesco Petrarca, Guarino Guarini und Gasparino Barzizza. ${ }^{9}$ In den folgenden Jahren wurde dieser Grundstock einer humanistischen Bibliothek etwa noch um Ausgaben von Livius, Gellius, Plutarch, Sueton und Plinius, Francesco Filelfos pulchrum epistolare sowie einen Druck von Leonardo Brunis Epistolae familiares erweitert. ${ }^{10} \mathrm{Im}$ Jahr 1474 erwarb die Fakultät überdies venezianische Drucke von Werken des Aristoteles, und zwar in alter und neuer Übersetzung, mit der vom Dekan eigenhändig notierten Begründung, die Fakultät habe erkannt, eine neue Textgrundlage insbesondere für den Unterricht der Bakkalare zu benötigen, um auf diesem Weg aus den trüben Abwässern zu den klaren Quellen der Philosophen zurückkehren zu können (ex fecibus ad nitidos philosophorum fontes rediremus). ${ }^{11}$

Als kurze Zeit später ein Mitglied des Herzogskollegs aus unbekanntem Grund nach Rom reiste, wurde ihm zuvor durch die Artistenfakultät der Auftrag erteilt, bei dieser Gelegenheit vor Ort auch nach seltenen Büchern zu suchen: ab bonum facultatis velle inservire eidem in libris raris, sive scriptis sive impressis, emendis pro magistrorum studio maxime accomodis... sese obtulerat. ${ }^{12}$

Diese humanistischen Bücherkäufe boten den Wiener Universitätsangehörigen nicht nur die Möglichkeit zur Lektüre der antiken lateinischen Literatur bzw. humanistischer Werke zu Rhetorik und Grammatik, sondern stellten darüber hinaus auch die Basis für entsprechende Lehrveranstaltungen an der artistischen Fakultät dar. So fanden zeitgleich mit dem Aufbau dieser humanistischen Büchersammlung auch einschlägige Vorlesungen an der Rudolfina statt, wobei in diesem Zusammenhang häufig der eben erwähnte Wolfgang Hayden aus Wien (Dekanat 1467/68) bzw. Paul von Stockerau (Dekanat 1474) in Erscheinung traten. Ersterer las unter anderem über Werke von Cicero und Horaz, letzterer über Vergils Bucolica. ${ }^{13}$ Von 1469 bis 1482 finden wir an der Wiener Artistenfakultät eine beinahe

umfangreicher Literaturangaben vgl. zuletzt Jana Madlen SchütTE, Akten: Rektorats-, Senats- und Fakultätsakten, in: Jan-Hendryk de Boer - Marian Füssel - Maximilian Schuh (Hgg.), Universitäre Gelehrtenkultur vom 13.-16. Jahrhundert. Ein interdisziplinäres Quellen- und Methodenhandbuch, Stuttgart 2018, S. 39-50.

8 Zur Geschichte der Wiener Universitätsbibliotheken im Mittelalter siehe zuletzt Friedrich SiMADER, Wertvoller als Gold und Silber. Die mittelalterlichen Bibliotheken der Universität Wien, in: Heidrun Rosenberg - Michael Viktor Schwarz (Hgg.), Wien 1365. Eine Universität entsteht, Wien 2015, S. 92-105. Zu Handschriften und Inkunabeln als Ausdruck der Aneignung des Humanismus im universitären Kontext siehe allgemein Maximilian Schuн, Aneignungen des Humanismus. Institutionelle und individuelle Praktiken an der Universität Ingolstadt im 15. Jahrhundert (Education and Society in the Middle Ages and Renaissance 47), Leiden-Boston 2013, S. 152-159.

9 Wien, Universitätsarchiv, Acta Facultatis Artium 3, Fol. 190v-191r.

10 Ebd. Fol. 206r, 214r, 245v und 247r.

11 Ebd. Fol. 245v.

12 Ebd. Fol. 248r.

13 Ebd. Fol. 199r, 206v, 221rv, 231v, 244r und 252r bzw. Fol. 252v. Wolfgang Hayden entstammt wohl einer einflussreichen Wiener Ratsfamilie, die überdies als Marktrichter in Perchtoldsdorf belegt ist; siehe Richard 
ununterbrochene Reihe humanistischer Vorlesungen, wobei neben den bereits Genannten auch ein gewisser Benedikt Kneysl aus Innsbruck, Sigmund von Schärding und Georg Pattersdorf aus Wasserburg am Inn als Vortragende in Erscheinung treten. ${ }^{14}$ Über Karriere und Bildungshorizont dieser nordalpinen Gelehrten ist wenig bekannt, zweifellos brachten sie jedoch der humanistischen Bildungsbewegung allgemeines Interesse entgegen, wohl ohne selbst in diesem Geiste literarisch aktiv zu werden.

Unklar muss in diesem Zusammenhang auch bleiben, in welchem Verhältnis diese Gruppe zu dem italienischen Franziskaner und Humanisten Lorenzo Traversagni da Savona steht, der zuvor in Bologna und Padua studiert hatte und ab dem Jahr 1452 in Wien nachweisbar ist. An der Rudolfina hielt Traversagni am 23. Juni 1453 eine öffentliche Disputation über Rhetorik, verfasste unter anderem einen humanistischen Modus epistolandi und konnte offenbar sehr rasch einen Kreis von interessierten Wiener Gelehrten um sich sammeln, zu dem unter anderem der insbesondere als Astronom bekannte Georg von Peuerbach gehörte. ${ }^{15}$ Diese Gruppe drängte die Wiener Universität schließlich dazu, für den Italiener einen Lehrstuhl in den studia humanitatis zu schaffen, scheiterte letztendlich wohl vor allem an mangelnder Unterstützung durch den Landesfürsten. ${ }^{16}$

Ein bislang kaum beachteter Schüler Traversagnis sollte später jedoch in eine politisch einflussreiche Position gelangen und für die Entwicklung des Humanismus eine durchaus bedeutsame Rolle einnehmen. Der Wiener Bürgersohn Johannes Rehwein wurde 1445 an der Rudolfina immatrikuliert, erreichte fünf Jahre später das artistische Lizentiat und absolvierte im Anschluss - ganz nach dem Vorbild seines Lehrers Traversagni - ein Studium der Rechte in Padua, wo er unter anderem den schwäbischen Humanisten Johannes Roth, einen Schüler Lorenzo Vallas, kennenlernte. Nach seiner Rückkehr blieb Rehwein den humanistischen Bildungsinhalten verbunden und versuchte sich etwa an poetischen Grußworten, die er im Zuge des kaiserlichen Adventus in Wien an Eleonore, die Gemahlin Kaiser Friedrichs III., richtete. ${ }^{17}$

$\mathrm{Ab}$ den 1460er Jahren ist Johannes Rehwein schließlich am Hof Kaiser Friedrichs III. als Teil eines humanistisch interessierten „Triumvirats“ tätig, zu dem neben Rehwein und dem eben genannten Johannes Roth noch ein weiterer Paduaner Kommilitone des Wieners gehörte, nämlich der Untersteirer Thomas Prelokar von Cilli, der später als erster humanistischer Praeceptor des jungen Thronfolgers Maximilian tätig wurde. ${ }^{18}$ Über ihre Kernaufgaben im habsburgischen Kanzleidienst hinaus wirkten diese Gelehrten aktiv für

Perger, Die Wiener Ratsbürger 1396-1526. Ein Handbuch, Wien 1988, S. 207f. (Forschungen und Beiträge zur Wiener Stadtgeschichte 18); Heinrich Demelius, Erhart Haidem, Richter zu Perchtoldsdorf bei Wien, und die landesfürstliche Gerichtsbarkeit. Ein Beitrag zur österreichischen Privatrechtsgeschichte des Spätmittelalters, Wien 1974 (Sitzungsberichte der philosophisch-historischen Klasse 293,1).

14 Siehe dazu K. Grossmann, Frühzeit, S. 259-261.

15 Siehe zuletzt Helmuth Grössing (Hg.), Der die Sterne liebte. Georg von Peuerbach und seine Zeit, Wien 2002; M. Wagendorfer, Eneas, S. 25-28, sowie Alfred Noe, Geschichte der italienischen Literatur in Österreich, I, Von den Anfängen bis 1797, Wien - Köln - Weimar 2011, S. $58 \mathrm{f}$.

16 K. Grossmann, Frühzeit, S. 229-232.

$17 \mathrm{Zu}$ Johannes Rehwein siehe ausführlich Daniel Luger, Humanismus und humanistische Schrift in der Kanzlei Kaiser Friedrichs III. (1440-1493), Wien - Köln - Weimar 2016, S. 97-110 (Mitteilungen des Instituts für Österreichische Geschichtsforschung, Ergänzungsband 60).

18 Siehe Alphons Lнотsкy, Die Wiener Artistenfakultät 1365-1497, Österreichische Akademie der Wissenschaften, Philosophisch-historische Klasse, Sitzungsberichte 247. Band, 2. Abteilung, Wien 1965, S. 170, bzw. D. Luger, Humanismus, s. 110-119. 
die Etablierung des Humanismus, zunächst im näheren Umfeld des kaiserlichen Hofes, in weiterer Folge auch an der Universität Wien. So ist etwa 1477/78 eine kaiserliche Gesandtschaft nach Venedig unter der Leitung von Thomas von Cilli belegt, die über Verhandlungen mit der Serenissima hinaus auch den ausdrücklichen Auftrag hatte, in Italien einen Gelehrten für den Dienst in der kaiserlichen Kanzlei zu rekrutieren. Auf einem Schiff von Triest nach Venedig sollen die habsburgischen Gesandten schließlich mit dem jungen Humanisten Pietro Bonomo in Kontakt getreten sein, während sich dieser - wie Bonomo später selbst berichtet - die Überfahrt mit der Lektüre von Plinius verkürzen wollte. ${ }^{19}$

Tatsächlich trat der Triestiner kurz darauf als Kanzlist und Diplomat in den Dienst Friedrichs III. ein. Bonomo blieb über viele Jahrzehnte am habsburgischen Hof tätig und war selbst noch unter den Nachfolgern Friedrichs III., als Rat Maximilians und Karls V. wie als Großkanzler Erzherzog Ferdinands, aktiv. In dieser Zeit verfasste der Italiener u.a. kleinere poetisch-panegyrische Werke und stand in engem Kontakt mit vor allem deutschsprachigen Humanisten. ${ }^{20}$

Wenige Jahre nach Bonomo wurde sicherlich auf Betreiben dieses höfischen Humanistenkreises mit dem Steirer Bernhard Perger ein weiterer humanistisch gebildeter Gelehrter in den kaiserlichen Hofdienst aufgenommen, der in weiterer Folge gemeinsam mit seinem Triestiner Kanzleikollegen eine bedeutende Rolle bei der Etablierung dieser Bildungsbewegung auch an der Artistenfakultät der Wiener Rudolfina einnehmen sollte.

Bernhard Perger studierte ab 1459 an der Wiener Rudolfina, auch er entschied sich daraufhin für einen Studienaufenthalt in Italien, von dem er spätestens im Frühjahr 1475 nach Wien zurückkehrte. Hier hielt er unter anderem Vorlesungen zu Vergil, Sallust, Horaz und Boethius, amtierte im Jahr 1478 als Dekan der Artistenfakultät und im Anschluss daran als Rektor. Zwei Jahre davor hatte Perger ein Studium an der juridischen Fakultät begonnen, an der er 1478 das Bakkalariat und 1481 das Lizentiat im kanonischen Recht erwarb. Außerdem war der Steirer als Schulmeister zu St. Stefan tätig. In diese Phase fällt auch die Entstehung von Pergers philologischem Hauptwerk, der vermutlich im Jahr 1479 entstandenen und kurz danach in Venedig gedruckten Grammatica Nova. Dabei handelt es sich um die erste lateinische Grammatik eines deutschsprachigen Humanisten, wobei sich Perger die Rudimenta des Niccolò Perotti zum Vorbild nahm und für deutschsprachige Leser adaptierte. Seine Grammatik war höchst erfolgreich und erlebte bis 1518 zumindest 36 Auflagen. ${ }^{21}$

19 Vgl. Stefano di Brazzano, Girolamo Muzio e Pietro Bonomo (Agosto 1517-Gennaio 1519), Atti e Memorie della Società Istriana di Archeologia e Storia Patria 99, 1999, S. 93-137, und D. Luger, Humanismus, S. 123.

$20 \mathrm{Zu}$ Pietro Bonomos Karriere siehe allgemein Stefano di Brazzano, Pietro Bonomo (1458-1546). Diplomatico, umanista e vescovo di Trieste. La vita e l'opera letteraria, Triest 2005 (Hesperides - Letterature e Culture Occidentali, Serie Gold 2), bzw. D. Luger, Humanismus, S. 119-132.

21 Siehe Cornelia Faustmann - Daniel Luger, Humanist und Naturwissenschaftler? Bernhard Perger zwischen Kanzleihumanismus, griechischer Philologie und dem Erbe Georgs von Peuerbach, in: Helmut Grössing Kurt Mühlberger (Hgg.), Wissenschaft und Kultur an der Zeitenwende. Renaissance-Humanismus, Naturwissenschaften und universitärer Alltag im 15. und 16. Jahrhundert, Göttingen 2012, S. 129-155; Hans-Christian KLuPaK, Personalbibliographien des Lehrkörpers der Wiener Artistenfakultät der Zeit von 1450 bis 1545 mit biographischen Angaben, Diss. Erlangen-Nürnberg 1974, S. 115-119, bzw. D. LugER, Humanismus, S. 132-149. 
Im Jahr 1481 war Bernhard Perger noch als Gesandter der Artistenfakultät an den päpstlichen Legaten Alexander Numai, Bischof von Forlì, tätig, ${ }^{22}$ im darauffolgenden Jahr jedoch verließ Perger die Universität, sicherlich im Zusammenhang mit dem gleichzeitigen Ausbruch der schweren militärischen Auseinandersetzungen zwischen Kaiser Friedrich III. und König Matthias Corvinus von Ungarn, die schließlich in der Belagerung und Einnahme Wiens durch ungarische Truppen gipfelten und auch der Rudolfina große wirtschaftliche Nöte einbrachten. ${ }^{23}$

Am habsburgischen Hof war der Steirer über seine Aufgaben in der erbländischen Kanzlei hinaus - wie die meisten seiner humanistisch gebildeten Kanzleikollegen - auch als kaiserlicher Diplomat tätig. Seiner universitären Ausbildung in Italien entsprechend lag Pergers Schwerpunkt in beiden Tätigkeitsbereichen auf den politischen Verhältnissen der Apenninhalbinsel. So wurde er beispielsweise im Jahr 1488 als kaiserlicher Kommissar in das habsburgische Pordenone entsandt und schloss bei dieser Gelegenheit engere Bekanntschaft mit dem dortigen Literatenzirkel um Paulus Amaltheus. ${ }^{24}$

Im Juni 1492 wurde Perger zum kaiserlichen Stadtanwalt in Wien - dem landesfürstlichen Vertreter im Wiener Stadtrat - ernannt, nahm in dieser Funktion an Ratssitzungen teil und sollte Beschlüsse, die gegen die Interessen des habsburgischen Stadtherrn gerichtet waren, verhindern. ${ }^{25}$ Die Universität Wien bat den Kaiser um die Einsetzung eines landesfürstlichen Superintendenten, wohl in der Hoffnung, auf diesem Weg eine einflussreiche Ansprechperson bei Hofe mit guten Verbindungen zum Landesfürsten zu gewinnen. In erster Linie ging es dabei vermutlich um die stockenden Einnahmen der Universität aus der landesfürstlichen Maut zu Ybbs zur Deckung von Stipendien. Deren Auszahlung geriet nach dem Ende der ungarischen Besatzungszeit ins Stocken, da der Kaiser der Universität Illoyalität während des vorangegangenen Konfliktes mit König Matthias von Ungarn vorwarf. Letztendlich ernannte der Habsburger jedoch Bernhard Perger auch zum Superintendenten der Universität, welcher dem Rektor bald vermelden konnte, den Kaiser zur Auszahlung entsprechender Gelder an die Universität bewegt zu haben. ${ }^{26}$

Als Superindentent der Universität beließ es Perger allerdings nicht bei der traditionellen Rolle eines Intervenienten, sondern nutzte seine einflussreiche Stellung für Eingriffe in das Selbstbestimmungsrecht der Universität, über die uns die Akten der artistischen Fakultät umfassend Aufschluss geben. Dabei ging es Perger zunächst um eine Reform des Studienbetriebes an der artistischen Fakultät, ganz im Sinne humanistischer Forderungen nach einem Ende der als nutzlos charakterisierten dialektischen Übungen sowie nach

22 Wien, Universitätsarchiv, Acta Facultatis Artium 3, Fol. 293r. Zu Bischof Alexander Numai als Legat in Österreich siehe Ferdinand Opll, Das Archiv eines päpstlichen Legaten aus der zweiten Hälfte des 15. Jahrhunderts. Ein Bestand im Wiener Stadt-und Landesarchiv, in: Rudolf Holbach - Michel Pauly (Hgg.), Städtische Wirtschaft im Mittelalter. Festschrift für Franz Irsigler zum 70. Geburtstag, Köln - Weimar - Wien 2011, S. 179-210; Bernd Erfle, Alexander Numai, Bischof von Forli, als Diplomat in Diensten von Papst und Kaiser (1470-1483), Diss. Marburg 2003.

23 Siehe dazu A. Lhotsky, Artistenfakultät, S. 166.

24 D. Luger, Humanismus, S. 136-140. Zu Amaltheus, einem Schüler des Cimbriacus, siehe unten bzw. A. NoE, Literatur, S. $63 \mathrm{f}$.

25 Vgl. Helmuth Grössing, Die Wiener Stadtanwälte im Spätmittelalter, Jahrbuch des Vereins für Geschichte der Stadt Wien 26, 1970, S. 36-45.

26 D. Luger, Humanismus, S. 140-142. Zum Verhältnis der Universität Wien zu den Herzögen von Österreich im Spätmittelalter im Allgemeinen siehe Paul UIBLEIN, Die österreichischen Landesfürsten und die Wiener Universität im Mittelalter, Mitteilungen des Instituts für Österreichische Geschichtsforschung 72, 1964, S. $382-408$. 
einer Rückbesinnung auf Originaltexte anstelle der Beschränkung auf Kommentare und Hilfstexte. Perger übergab dem Dekan in der Fakultätssitzung vom 13. Oktober 1492 eine Liste seiner diesbezüglichen Forderungen (desideria). Die darauffolgende Reaktion der Fakultät klingt durchaus modern - sie setzte nämlich eine Reformkommission ein, über deren Tätigkeit keinerlei Quellen vorliegen..$^{27}$ Der euphorische Bericht des humanistisch interessierten Wiener Arztes Bartholomäus Stäber (Scipio) an Konrad Celtis, wonach die Universität Wien nun mit Willen des Kaisers von Dornen und Gestrüpp gereinigt werde und den humanistischen Studien nun ein besonderer Platz unter den artes zukommen werde, ${ }^{28}$ muss jedenfalls als voreilig gelten. Jedenfalls beschloss die Fakultät am 17. Juni 1493, den Studenten eine Empfehlung zur gründlicheren Beschäftigung mit den Originaltexten auszusprechen. In dieser Sitzung berichtet der Dekan auch über eine weitere Idee Pergers, nämlich die regelmäßige Abhaltung von conversationes plateales, d.h. von öffentlichen Zusammenkünften von Lehrern und Schülern unter freiem Himmel. Diese sollten in erster Linie zu wissenschaftlichen Besprechungen genutzt werden und vor dem nahen Dominikanerkloster stattfindenstattfinden. Nach dem Bericht des Dekans seien bei diesen Gelegenheiten jedoch vielmehr die schlechten Sitten so mancher Teilnehmer an den Tag getreten, weshalb sich die Wiener Bürgerschaft über diese Neuerung beschwert habe. Daher wurde diese Initiative Pergers bereits wenige Monate später wieder wieder aufgegeben, wobei als Vorwand die früh eintretende Dunkelheit (propter advenientes tenebras noctis) herangezogen wurde. ${ }^{29}$

Die von Perger angestrebte Reform des universitären Studiums sollte von einer personellen Erneuerung begleitet werden. Der bayrische Humanist und Sekretär Kaiser Friedrichs III. Johann Krachenberger vermeldete an Konrad Celtis, der selbst an einer humanistischen Lektur in Wien interessiert war, dass Pergers Fokus ganz auf der Berufung von Humanisten aus Italien läge. Dies brachte Perger schließlich das bekannte, wütende Epigramm des verschmähten Celtis mit dem Titel De Perger, scriba Caesareo ein. ${ }^{30}$ Nach Celtis vertrete Perger die Ansicht, wonach nur Italien über gelehrte Dichter verfüge, von deutschen Musen hingegen wolle er gar nichts wissen (Germanas non vult cognoscere Musas). Zwar habe Celtis das Ohr des Superintendenten bereits drei oder vier Mal voller Demut bestürmt, dieser aber zeige seinen Gedichten und Werken die Nase und bevorzuge kleine italienische Poeten. Celtis' Anklage gipfelte schließlich in den beiden invektiven Versen: Non te Germana iam dicam stirpe creatum, dum spernis patriam, perfide Slave, meam.

Tatsächlich versuchte Perger offenbar seit Beginn seiner Tätigkeit als Superintendent, aus Italien kommende Humanisten an die Wiener Rudolfina zu berufen. Den Anfang machte der Triestiner Francesco Bonomo, ein Bruder von Pergers Kanzleikollegen Pietro Bonomo, der Steirer für die poetischen Lektur in Wien gewinnen wollte. Krachenberger vermeldete im Februar 1493 an Celtis, dass der Italiener jeden Tag zu seiner lectura in Wien erwartet werde. ${ }^{31}$ Aus unbekannten Gründen scheint es allerdings nie zu einer Berufung Bonomos gekommen zu sein.

\footnotetext{
Wien, Universitätsarchiv, Acta Facultatis Artium 3, Fol. 357r.

Zitat nach: K. Grossmann, Frühzeit, S. 301.

Wien, Universitätsarchiv, Acta Facultatis Artium 3, Fol. 362r+v.

30 Ediert in: Gustav Bauch, Die Reception des Humanismus in Wien. Eine literarische Studie zur deutschen Universitätsgeschichte, Breslau 1903, S. 58.

31 K. Grossmann, Frühzeit, S. 300.
} 
Pergers nächster Kandidat war der bereits oben genannte Paulus Amaltheus aus Pordenone, der im Mai 1493 auf ausdrücklichen Wunsch des Superintendenten als Lehrer der Poesie und Rhetorik an die Universität Wien berufen wurde. Amaltheus sollte für ein von der Universität bezogenes jährliches Stipendium von 50 Gulden zwei Vorlesungen täglich abhalten. Laut den Akten der artistischen Fakultät erschien dem Italiener diese Bedingung jedoch unannehmbar; er war unter diesen Umständen nur zur Abhaltung einer Vorlesung täglich bereit. Diese Angelegenheit blieb ein ständiger Konfliktherd und gelangte schließlich an den Superintendenten. Die Fakultät drängte den Italiener hartnäckig zur Abhaltung einer zweiten poetischen Vorlesung, da Amaltheus jedoch weiterhin nicht dazu bereit war, einigte man sich schließlich auf die Auszahlung einer Teilsumme des Stipendiums und den vorzeitigen Abschied des Italieners mit Ende September 1493. De aliis silendum est ex honesto, wird vom Dekan in den Fakultätsakten dazu lapidar vermerkt. ${ }^{32}$

Durch diesen Mißerfolg ließ sich der Superintendent Perger jedoch nicht entmutigen und berief - wohl auf Vermittlung des ungarischen Humanisten Johann Vitéz, Bischof von Veszprém und Administrator des Bistums Wien - als Nachfolger des Amaltheus einen weiteren Italiener, nämlich den Venezianer Hieronymus Balbus (Girolamo Balbi) nach Wien. Balbus hatte zuvor in Paris Vorlesungen über Poesie gehalten; dort sei er jedoch für außergewöhnlich hochmütig gehalten worden, auch sein Lebenswandel habe ihm Kritik eingebracht. ${ }^{33}$ In Wien jedenfalls ließ sich Balbus im Sommersemester 1493 immatrikulieren, sein Eintrag in der juridischen Matrikel wurde jedoch mit dem vielsagenden Zusatz ergänzt: a principe missus. ${ }^{34}$ Der Italiener hielt sich zunächst weiterhin im Umfeld des Johann Vitez in Ungarn auf; am 22. Juni 1494 übernahm Balbus schließlich den neugegründeten Lehrstuhl für römisches Recht in Wien und erhielt im selben Jahr zusätzlich die erste eigentliche poetische Lektur an der Rudolfina mit fixer, direkt von Landesfürsten bezogener Besoldung. Allerdings kam es auch in diesem Fall zu Streitigkeiten zwischen dem Italiener und der artistischen Fakultät um den von Balbus geforderten verpflichtenden Besuch seiner Vorlesungen sowie erneut um Fragen der Besoldung, da Balbus neben seinem Gehalt auch universitäre Kollegiengelder beziehen wollte. Auf Druck der landesfürstlichen Regenten verzichtete der Italiener schließlich auf Teile seiner Forderung und setzte seine Lehrtätigkeit fort, hatte in weiterer Folge jedoch mit Kritik an der Qualität seiner Vorlesungen zu kämpfen: qualiter tamen legit, sciunt isti, qui audiverunt, wie es in den Fakultätsakten dazu maliziös heißt. ${ }^{35}$ Balbus verlor schließlich im Zuge dieses schwelenden Konfliktes mit der Fakultät die Unterstützung des Superintendenten, trat im Jahr 1497 von seiner Professur zurück und verließ bald darauf Wien, um später an der Universität Prag zu unterrichten. ${ }^{36}$

32 Wien, Universitätsarchiv, Acta Facultatis Artium 3, Fol. 363r. Zu Amaltheus siehe H. Grössing, Universität, S. 42; A. LнотSку, Artistenfakultät, S. 191-194; Herwig Schlögl, Lateinische Hofpoesie unter Maximilian I., Diss. Wien 1969, s. 89-118; bzw. A. NoE, Geschichte, 63f.

33 A. Noe, Literatur, S. 64; A. Lhotsky, Artistenfakultät, S. 194-97; K. Grossmann, Frühzeit, S. 291-294; H. Schlögl, Hofpoesie, S. 119-173.

34 Thomas Maisel - Johannes SeIDL - Severin Matiasovits (Hgg.), Die Matrikel der Wiener Rechtswissenschaftlichen Fakultät. Matricula Facultatis Juristarum. Studii Wiennensis, II, 1442-1557, bearbeitet von Severin Matiasovits, Wien 2016 (Publikationen des Instituts für Österreichische Geschichtsforschung, VI. Reihe: Quellen zur Geschichte der Universität Wien, 3. Abt.), S. 56: Dominus Ieronimus Balbus bonarum arcium atque utriusque iuris interpres fundatissimus pro eo, quod a principe missus, dedit $60 \mathrm{~d}$.

35 Wien, Universitätsarchiv, Acta Facultatis Artium 3, fol. 370v.

36 K. Grossmann, Frühzeit, S. 293. 
Nach dieser missglückten Personalpolitik Bernhard Pergers übernahm im Herbst des Jahres 1497 schließlich mit dem Franken Konrad Celtis ein deutschsprachiger Humanist die poetische Lektur in Wien. Die universitätsgeschichtliche Forschung hat die Berufung dieses nordalpinen Humanisten mit einem Machtverlust Pergers begründet, der schlussendlich den landesfürstlichen Regenten Fuchsmagen und Krachenberger - beide ausdrückliche Befürworter des Celtis - unterlegen sei. ${ }^{37}$ Allerdings hängt diese Entwicklung mit dem frühen Ableben Pergers zusammen, denn dieser verstarb nicht, wie bislang vermutet, erst nach der Übernahme der Superintendenz durch Cuspinian im Jahr 1501, ${ }^{38}$ sondern noch vor Celtis' Ankunft in Wien im Sommer des Jahres 1497, wie die jüngst im Archiv des Wiener Schottenstiftes aufgefunden Akten zu Pergers Verlassenschaft eindeutig belegen. ${ }^{39}$ Auch wenn man Bernhard Perger somit nicht mehr mit der letztendlich erfolgreichen humanistischen Studienreform des Jahres 1499 in Verbindung bringen kann, ${ }^{40}$ muss der Steirer sicherlich dennoch als einer der zentralen Wegbereiter des Humanismus an der Universität Wien wie auch am habsburgischen Hof gelten.

Unter diesem Blickwinkel gilt die Regierungszeit Kaiser Maximilians I. sicherlich zu Recht als Blütezeit des Humanismus in Österreich. Diese erschien jedoch nicht ex nihilo, sondern konnte auf ausgeprägte Vorformen aufbauen, die sich unter Maximilians Vorgänger und Vater entwickelten. Kaiser Friedrich III. nahm persönlich wohl kaum Anteil an dieser neuen Bildungsbewegung, für die ideologische Nutzbarkeit des Humanismus scheint er jedoch durchaus aufgeschlossen gewesen zu sein. So begegnen etwa humanistische Argumentationsmotive im Diktat kaiserlicher Urkunden, besonders augenfällig ist jedoch der Einsatz humanistischer Gelehrsamkeit auf dem bislang weniger beachteten Gebiet der Epigraphik. Wie etwa am Beispiel einer antikisierenden Inschrift aus Triest deutlich wird, lässt sich bereits Kaiser Friedrich III. von seinen Hofhumanisten als Nachfolger der antiken römischen Imperatoren stilisieren. So wurde anlässlich einer vermutlich im Jahr 1483 erfolgten Renovierung der Triestiner Stadtbefestigung vor Ort eine Bauinschrift angebracht, welche die Kopie einer noch heute im Original überlieferten Inschrift von Oktavian aus dem Jahr 32 v. Chr. darstellt. Sicherlich auf Betreiben seines epigraphisch interessierten Triestiner Hofhumanisten Pietro Bonomo ließ Kaiser Friedrich III. den antiken Inschriftentext jedoch um zwei weitere Zeilen erweitern: FRI(DERICUS) TER(TIUS) RO(MANORUM) IMP(ERATOR) DUX AUST(RIAE) (ET) C(E)T(ERA) DO(MINUS)Q(UE) TER/GESTI IIII VICE MUR[UM REEDI]FICARI IUSSIT ${ }^{41}$

37 So K. Grossmann, Frühzeit, S. 312. Zu Konrad Celtis an der Rudolfina vgl. insbesondere Helmuth GrösSING, Die Lehrtätigkeit des Konrad Celtis in Wien. Ein Rekonstruktionsversuch, in: Kurt Mühlberger - Meta Niederkorn-Bruck (Hgg.), Die Universität Wien im Konzert europäischer Bildungszentren 14.-16. Jahrhundert, Wien - München 2010, S. 223-233 (Veröffentlichungen des Instituts für Österreichische Geschichtsforschung 56); Elisabeth KLECKER, Das Reich der Gelehrten. Europa im Blick der Wiener Humanisten um 1500, in: Heidrun Rosenberg - Michael Viktor Schwarz (Hgg.), Wien 1365. Eine Universität entsteht, Wien 2015, S. 250-265.

38 So bereits J. AschBACH, Geschichte, I, S. $575 \mathrm{f}$.

39 Siehe D. Luger, Humanismus, S. $142 \mathrm{f}$.

40 Irrig: K. Grossmann, Frühzeit, S. 302.

41 Siehe dazu Ortolf HARL - Alois NiedERSTÄTtER, Kaiser Friedrich III. als Nachfolger Caesars: Zwei Inschriften zur Befestigung von Tergeste/Triest, in: Beruf(ung): Archivar. Festschrift für Lorenz Mikoletzky, hg. von der Generaldirektion des Österreichischen Staatsarchivs, S. 699-725 (Mitteilungen des Österreichischen Staatsarchivs 55, Wien 2011). 
Im engen Zusammenspiel mit dem Humanismus am habsburgischen Hof muss auch die Entwicklung an der Universität Wien betrachtet werden, wo in der zweiten Hälfte des 15. Jahrhunderts zunehmend humanistisch gebildete, nordalpine Magister und Studenten auftraten, die dieser neuen Geisteshaltung möglicherweise vielfach oberflächlich, doch aber mit grundsätzlichem Interesse begegneten. Auch die Akten der artistischen Fakultät zeigen eine gewisse Offenheit gegenüber humanistischen Studien, wie etwa der Erwerb einschlägiger Handschriften und Drucke für die Universitätsbibliothek belegt. Humanistische Vorlesungen wurden seitens der Fakultät nicht nur geduldet, sondern - wie der Fall Amaltheus deutlich macht - sogar ausdrücklich eingefordert. Insbesondere die poetischen Lektionen des Balbus sollen laut einem Bericht des Dekans auch ausgesprochen gut besucht gewesen sein. Bei näherer Durchsicht des spätmittelalterlichen Aktenmaterials, das bislang leider nur in Teilen gedruckt ist, verschwimmen daher die bislang häufig gezeichneten Konfliktlinien zwischen einer scheinbar konservativen Artistenfakultät und den „modernen“ humanistischen Poeten, aber auch zwischen den Vertretern eines vermeintlich italienischen bzw. deutschen Humanismus. Selbst wenn die Reformversuche Pergers so manchen Universitätsangehörigen zu weit gingen und dessen Vorliebe für die Berufung italienischer Gelehrter die Beliebtheit dieser Anliegen an der Universität wohl nicht gerade steigerte, wird nach Durchsicht der Fakultätsakten deutlich, dass sich die Widerstände der artistischen Fakultät nicht grundsätzlich gegen das humanistische Bildungsprogramm richteten, sondern vielmehr gegen obrigkeitliche Eingriffe in die universitäre Selbstverwaltung.

\section{DANIEL LUGER}

\section{Italští humanisté na vídeňské univerzitě v 15 . století - mezi podporou a odmítnutím}

\section{RESUMÉ}

V centru pozornosti tohoto př́spěvku stojí humanistický reformní program na vídeňské univerzitě ve druhé polovině 15. století. Ústřední roli přitom hraje dvorský učenecký kroužek, jemuž se dosud dostalo malé pozornosti. Protonotář Bernhard Perger může být označen jako iniciátor prvního humanistického reformního programu, který obsahoval jak univerzitní učební provoz, tak personální obměnu učitelského kolektivu. Perger se opíral o italské učence, kteří ve Vídni záhy vyvolali opakované konflikty. Ty se netýkaly rozdílů v obsahu výuky na artistické fakultě. Po prostudování univerzitního aktového materiálu je zřejmé, že artistická fakulta záhy projevovala otevřenost vůči humanistickým studiím, jak dokládají např. rukopisy a tisky získané pro univerzitní knihovnu. Humanistické přednášky byly ze strany fakulty nejen trpěny, ale i výslovně vyžadovány. Dosud často zmiňované konfliktní situace proto musejí být nově zváženy. 